Tropical Journal of Pharmaceutical Research October 2018; 17 (10): 2055-2060

ISSN: $1596-5996$ (print); 1596-9827 (electronic)

(c) Pharmacotherapy Group, Faculty of Pharmacy, University of Benin, Benin City, 300001 Nigeria.

Original Research Article

http://dx.doi.org/10.4314/tjpr.v17i10.23

\title{
Online quality control of panaxatriol saponins percolation extraction using near-infrared technology
}

\author{
Rui-xue Zhong ${ }^{1}$, Yu-jie Liu ${ }^{2}$, Jun Wan ${ }^{3}$, Li-yang Zhu', Yun-bei Chen ${ }^{1}$, Hui-min \\ Wen $^{1}$, Xiao-yun Duan ${ }^{1}$, Xiao-ling Wang ${ }^{1}$, Ting-sen $\mathrm{Wei}^{1}$, Chuan-hong $\mathrm{Wu}^{1}$, \\ Guang-fu Feng ${ }^{1 *}$ \\ ${ }^{1}$ Department of Pharmacy, Sichuan Provincial Orthopedic Hospital, Chengdu 610041, ${ }^{2}$ School of Pharmacy, Chengdu Medical \\ College, Chengdu 610500, ${ }^{3}$ Life Science \& Engineering College of South-west Jiao-tong University, Chengdu 610031, PR \\ China
}

${ }^{*}$ For correspondence: Email: fengguangfu65@163.com; Tel: +86-18908188545

Sent for review: 25 May 2018

Revised accepted: 26 September 2018

\begin{abstract}
Purpose: To establish a new prediction model for online quality control of the percolation extraction of panaxatriol saponins (PTS), viz, ginsenoside Rg1, ginsenoside Re and notoginsenoside R1, from notoginseng by near-infrared (NIR) technology coupled with partial least squares (PLS) analysis.

Methods: Ten batches of PTS (420 samples) were collected and the constituents were determined using HPLC. The NIR spectroscopy of samples was determined using a Fourier-Transform nearinfrared spectrometer with an optical fiber transmission PbS detector. Eight sample batches were the calibration set, and two batches were the forecast set. Calibration models were established based on min-max normalization (MMN).

Results: The root mean square errors of cross-validation (RMSECV) of Rg1, Re, and R1 were 0.798 , 0.095 , and $0.259 \mathrm{mg} / \mathrm{mL}$, respectively. The root mean square errors of prediction (RMSEP) were 1.110 , 0.496 , and $0.390 \mathrm{mg} / \mathrm{mL}$, respectively. The correlation coefficients $\left(R^{2}\right)$ of cross-validation were 0.9682 , 0.9681 , and 0.9626 , respectively, while the correlation coefficients $\left(R^{2}\right)$ of prediction were 0.9831 , 0.9198 , and 0.9661, respectively.

Conclusion: The results indicate that NIR is a quick and effective tool for online quality control of PTS (ginsenoside Rg1, ginsenoside Re, and notoginsenoside R1) in the percolation extraction process.
\end{abstract}

Keywords: Online monitoring, Near infrared technology, Panaxatriol saponins, Partial least squares

\begin{abstract}
This is an Open Access article that uses a funding model which does not charge readers or their institutions for access and distributed under the terms of the Creative Commons Attribution License (http://creativecommons.org/licenses/by/4.0) and the Budapest Open Access Initiative (http://www.budapestopenaccessinitiative.org/read), which permit unrestricted use, distribution, and reproduction in any medium, provided the original work is properly credited.

Tropical Journal of Pharmaceutical Research is indexed by Science Citation Index (SciSearch), Scopus, International Pharmaceutical Abstract, Chemical Abstracts, Embase, Index Copernicus, EBSCO, African Index Medicus, JournalSeek, Journal Citation Reports/Science Edition, Directory of Open Access Journals (DOAJ), African Journal Online, Bioline International, Open-J-Gate and Pharmacy Abstracts
\end{abstract}

\section{INTRODUCTION}

Traditional Chinese Medicine (TCM) has gained worldwide popularity among researchers [1-3]. The root or rhizome of Panax notoginseng (Burk.) F. H. Chen (notoginseng) was first used as a medicinal plant in China because of its protective hemostatic and cardiovascular effects $[4,5]$. Panaxatriol saponins (PTS), the active constituents extracted from notoginseng, contains ginsenoside $\mathrm{Re}$, ginsenoside Rg1, and notoginsenoside R1. Pharmacological studies indicate that PTS is the main active constituent of notoginseng and is often used for quality control 
[6-8]. Percolation is the first step in the extraction of PTS from notoginseng, which directly influences PTS quality. Therefore, it is important to control the quality of PTS in the percolation process efficiently and rapidly. High-performance liquid chromatography (HPLC) is a useful tool for quality control of PTS in the percolation extraction process to determine the three active constituents [9-11]. However, this method is insufficient due to the hysteretic checking result and tedious sample preparation. Thus, a new method is needed that can instantly obtain results without pretreatment for the online quality control of the percolation extraction process.

In the present study, new mathematical models for PTS using near-infrared(NIR) combined with partial least squares (PLS) were established for the online quality control of the percolation extraction process, which helps to guarantee the PTS quality.

\section{EXPERIMENTAL}

\section{Chemicals and reagents}

Standards of ginsenoside Rg1, notoginsenoside $R 1$, and ginsenoside $R e$ were purchased from the National Institute for the Control of Pharmaceutical and Biological Products (Beijing, China). Chromatographic acetonitrile was purchased from Fisher Chemicals (Fair Lawn, New Jersey, USA). The ultrapure water used was purified using the Millipore purification system (Millipore, MA, USA). All other reagents used in this study were of analytical grade.

\section{Sample preparation}

Samples were powdered and strained through a standard 24 mesh sieve $(850 \pm 29 \mu \mathrm{m})$. The powder was first immersed in $60 \%$ ethanol for $24 \mathrm{~h}$ and then percolated with a flow rate of $5-8$ $\mathrm{mL} / \mathrm{min}$. The percolation process lasted for 19 and $25 \mathrm{~mL}$ samples were collected: $0-5 \mathrm{~h}$, collected every $1 \mathrm{~h} ; 5-15 \mathrm{~h}$, collected every 30 min; $15-19 \mathrm{~h}$, collected every $15 \mathrm{~min}$. A total of 420 samples were obtained (42 samples for each batch). Each sample was directly determined using a $\mathrm{PbS}$ detector at room temperature. Batches 1, 2, 3, 4, 6, 7, 8 and 9 were the calibration set, whereas batches 5 and 10 were the forecast set.

\section{HPLC chromatographic conditions}

HPLC was performed using an Agilent 1200 high-performance liquid chromatography (HPLC) system (Agilent Technologies, CA, USA). The samples were analyzed on an Agilent C18 column ( $250 \mathrm{~mm} \times 4.6 \mathrm{~mm}, 5 \mu \mathrm{m}$, Agilent). The elution system contained acetonitrile:water (19.5:80.5) at a $1.0 \mathrm{~mL} / \mathrm{min}$ flow rate and the column temperature was maintained at $30{ }^{\circ} \mathrm{C}$. The wavelength was set at $210 \mathrm{~nm}$.

\section{The near-infrared spectroscopy conditions}

The NIR spectroscopy was determined using a Bruker Matrix-I Fourier-Transform Near-Infrared (FT-NIR) spectrometer (Bruker Optik, Ettlingen, Germany). An optical fiber transmission PbS detector was used for spectra acquisition and the OPUS workstation was employed for dataprocessing. The absorbance of air was regarded as the reference standard. Each sample was scanned 64 times with the resolution of $8 \mathrm{~cm}^{-1}$ over the scan range from 12500 to $3600 \mathrm{~cm}^{-1}$. After the automatic baseline corrections of the FT-NIR, the samples were added in $10 \mathrm{~mL}$ beakers and the optical fiber transmission $\mathrm{PbS}$ detector was inserted. Every sample was scanned three times after the sample was stable without bubbles and the mean value was used for the final analysis.

\section{RESULTS}

\section{Saponin content of the samples}

The calibration set had 336 samples and the validation set had 84 samples. Table 1 shows the contents of notoginsenoside R1, ginsenoside $\mathrm{Rg} 1$, and ginsenoside Re during the percolation extraction process.

\section{Primary selection of the spectrum region}

In this study, PLS method was employed for analyzing the models. Although PLS can assess the whole spectrum, this increases computational cost and reduces predictive power. In Figure 1, the absorbance for the wavelength range 12000$8000 \mathrm{~cm}^{-1}$ was almost a flat line, whereas the absorbance for the wavelength range 8000-4000 $\mathrm{cm}^{-1}$ changed significantly. This suggests that the wavelength range of $8000-4000 \mathrm{~cm}^{-1}$ may contain abundant information, which could reflect the sample composition. Therefore, the wavelength range of $8000-4000 \mathrm{~cm}^{-1}$ was selected for analysis using PLS method.

\section{Selecting pretreatment methods}

The OPUS workstation was developed by Bruker Instruments LTD (Bruker Optik, Ettlingen, Germany) and has 11 complete spectral pretreatment methods including min-max normalization (MMN), vector normalization (VN), and constant offset elimination (COE). 
Table 1: Ginsenoside Rg1, notoginsenoside R1 and ginsenoside Re contents of PTS samples

\begin{tabular}{ccccccc}
\hline No. & $\begin{array}{c}\text { Notoginsenoside } \\
\mathbf{R} \mathbf{1}(\mathbf{m g} / \mathbf{m L})\end{array}$ & Mean \pm SD & $\begin{array}{c}\text { Ginsenoside } \\
\mathbf{R g} \mathbf{1}(\mathbf{m g} / \mathbf{m L})\end{array}$ & Mean \pm SD & $\begin{array}{c}\text { Ginsenoside } \\
\mathbf{R e}(\mathbf{m g} / \mathbf{m L})\end{array}$ & Mean \pm SD \\
\hline 1 & $0.009-5.647$ & $0.973 \pm 1.624$ & $0.026-20.768$ & $3.677 \pm 5.976$ & $0.007-2.612$ & $0.463 \pm 0.760$ \\
2 & $0.012-5.874$ & $1.002 \pm 1.586$ & $0.083-22.345$ & $4.897 \pm 5.654$ & $0.005-2.222$ & $0.383 \pm 0.670$ \\
3 & $0.008-5.534$ & $0.962 \pm 1.683$ & $0.102-24.156$ & $5.013 \pm 4.980$ & $0.011-3.192$ & $0.642 \pm 0.860$ \\
4 & $0.010-5.423$ & $0.978 \pm 1.658$ & $0.032-20.654$ & $3.572 \pm 5.576$ & $0.012-3.023$ & $0.584 \pm 0.579$ \\
5 & $0.014-5.987$ & $1.123 \pm 1.643$ & $0.028-21.678$ & $3.574 \pm 4.986$ & $0.003-2.012$ & $0.343 \pm 0.570$ \\
6 & $0.007-4.988$ & $0.875 \pm 1.567$ & $0.046-19.789$ & $3.321 \pm 5.076$ & $0.006-2.985$ & $0.547 \pm 0.589$ \\
7 & $0.009-5.547$ & $0.957 \pm 1.524$ & $0.031-20.789$ & $3.572 \pm 5.074$ & $0.004-3.041$ & $0.513 \pm 0.660$ \\
8 & $0.008-5.564$ & $0.945 \pm 1.663$ & $0.076-23.485$ & $3.134 \pm 5.345$ & $0.015-4.091$ & $0.768 \pm 0.660$ \\
9 & $0.013-5.864$ & $1.088 \pm 1.563$ & $0.045-20.473$ & $4.121 \pm 6.076$ & $0.007-2.721$ & $0.440 \pm 0.563$ \\
10 & $0.011-5.426$ & $1.002 \pm 1.513$ & $0.093-25.473$ & $5.413 \pm 6.125$ & $0.006-2.619$ & $0.742 \pm 0.689$ \\
\hline
\end{tabular}

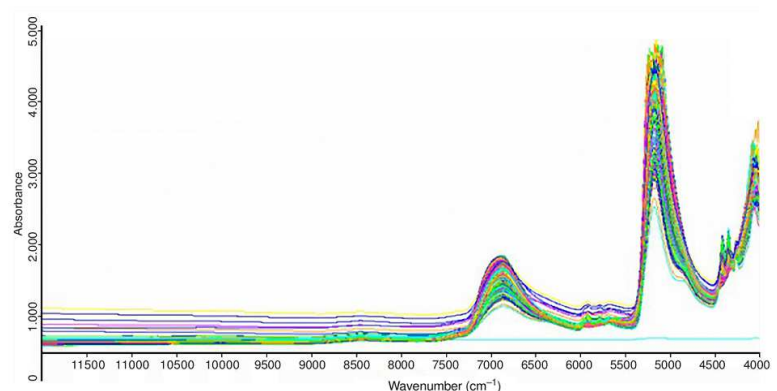

Figure 1: The NIR spectrum of the samples

From the OPUS, the predictive ability of different models was assessed for low root mean square errors of cross-validation (RMSECV) and root mean square errors of prediction (RMSEP) and high $\mathrm{R}^{2}$. As shown in Table 2 and Table 3, MMN was the most appropriate method for ginsenoside Rg1, ginsenoside Re, and notoginsenoside $\mathrm{R} 1$ analyses.

\section{Determination of the dimensions (D) of PLS factors}

The PLS $D$ values greatly affected the prediction results of the calibration models. Even in the same spectral pretreatment method, the $D$ value could directly influence the values of $R^{2}$, RMSECV, and RMSEP. A $D$ value that is too small could lead to insufficient spectral information, whereas a $D$ value that is too large could cause over fitting of the model. The OPUS could successfully recommend the optimal $D$ value. The statistical parameters versus $D$ are presented in Figure 2.

Table 2: Comparison of various pretreatment methods

\begin{tabular}{|c|c|c|c|c|c|c|c|}
\hline & \multirow{2}{*}{ Pretreatment } & \multirow{2}{*}{ Wavelength $\left(\mathrm{cm}^{-1}\right)$} & \multirow{2}{*}{ D } & \multicolumn{2}{|c|}{ Calibration set } & \multicolumn{2}{|c|}{ Validation set } \\
\hline & & & & $R^{2}$ & RMSECV & $R^{2}$ & RMSEP \\
\hline \multirow{10}{*}{ Ginsenoside Rg1 } & MMN & $\begin{array}{l}7501.9-5449.9 \\
4601.4-4246.5\end{array}$ & 19 & 0.9726 & 0.796 & 0.9737 & 1.423 \\
\hline & MMN & $\begin{array}{l}6475.9-5449.9 \\
4601.4-4246.5\end{array}$ & 17 & 0.9682 & 0.798 & 0.9831 & 1.110 \\
\hline & MMN & $\begin{array}{l}6101.8-5449.9 \\
4601.4-4246.4\end{array}$ & 17 & 0.9618 & 0.877 & 0.9664 & 1.458 \\
\hline & VN & $\begin{array}{l}7501.9-5449.9 \\
4601.4-4246.5\end{array}$ & 18 & 0.9679 & 0.774 & 0.9576 & 1.678 \\
\hline & MMN & $\begin{array}{c}11995.2-7498.0 \\
6101.8-5449.9 \\
4601.4-4246.4\end{array}$ & 19 & 0.9642 & 0.803 & 0.9395 & 2.006 \\
\hline & MMN & $\begin{array}{c}11995.2-5449.9 \\
4601.4-4246.5\end{array}$ & 20 & 0.9743 & 0.681 & 0.9616 & 1.626 \\
\hline & COE & $\begin{array}{l}6101.8-5449.9 \\
4601.4-4246.4\end{array}$ & 17 & 0.9606 & 0.842 & 0.9546 & 1.646 \\
\hline & MMN & $\begin{array}{l}6475.9-5449.9 \\
4601.4-4224.0\end{array}$ & 16 & 0.9608 & 0.796 & 0.9541 & 1.605 \\
\hline & MMN & $\begin{array}{l}7501.9-6475.9 \\
4601.4-4246.5\end{array}$ & 14 & 0.9291 & 0.598 & 0.9179 & 2.245 \\
\hline & MMN & $\begin{array}{l}6475.9-5449.9 \\
4601.4-4424.0 \\
\end{array}$ & 17 & 0.9607 & 0.428 & 0.9421 & 1.885 \\
\hline
\end{tabular}

MMN: min-max normalization; VN: vector normalization; COE: constant offset elimination; FD: First derivative; D: dimensions 
Table 3: Comparison of various pretreatment methods (contd)

\begin{tabular}{|c|c|c|c|c|c|c|c|}
\hline \multirow{3}{*}{ Ginsenoside Re } & MMN & $\begin{array}{l}6475.9-5449.9 \\
4601.4-4224.0\end{array}$ & 18 & 0.9681 & 0.095 & 0.9198 & 0.496 \\
\hline & $\mathrm{FD}+\mathrm{VN}$ & $\begin{array}{c}11995.2-6097.9 \\
4601.4-4246.5\end{array}$ & 18 & 0.9177 & 0.065 & 0.8746 & 0.571 \\
\hline & MMN & $\begin{array}{c}11995.2-7498.0 \\
6101.8-5449.9 \\
4601.4-4246.4 \\
\end{array}$ & 18 & 0.9194 & 0.061 & 0.8866 & 0.550 \\
\hline \multirow{6}{*}{$\begin{array}{l}\text { Notoginsenoside } \\
\text { R1 }\end{array}$} & MMN & $\begin{array}{l}7501.9-5449.9 \\
4601.4-4246.5\end{array}$ & 24 & 0.9626 & 0.259 & 0.9661 & 0.390 \\
\hline & VN & $\begin{array}{l}7501.9-5449.9 \\
4601.4-4246.5\end{array}$ & 20 & 0.9651 & 0.222 & 0.9334 & 0.598 \\
\hline & $\mathrm{FD}+\mathrm{VN}$ & $\begin{array}{c}11995.2-7498.0 \\
6101.8-5449.9 \\
4601.4-4246.4\end{array}$ & 13 & 0.9238 & 0.083 & 0.8477 & 0.972 \\
\hline & VN & $\begin{array}{l}6101.8-5449.9 \\
4601.4-4246.4\end{array}$ & 13 & 0.9453 & 0.049 & 0.8252 & 1.080 \\
\hline & MMN & $\begin{array}{l}7501.9-6097.7 \\
4601.4-4246.5\end{array}$ & 15 & 0.9687 & 0.037 & 0.8054 & 1.053 \\
\hline & VN & $\begin{array}{l}11995.2-5449.9 \\
4601.4-4246.5\end{array}$ & 14 & 0.9665 & 0.038 & 0.8248 & 1.062 \\
\hline
\end{tabular}

MMN: min-max normalization; VN: vector normalization; COE: constant offset elimination; FD: First Derivative; D: dimensions

As can be seen in Figure 2, when the $D$ value increased, the value of RMSECV decreased, but $\mathrm{R}^{2}$ increased in the calibration sets. Moreover, the two parameters were stable when the $D$ value achieved a certain value. These $D$ values for ginsenoside Rg1, ginsenoside $R e$, and notoginsenoside R1 acquired by OPUS were 17, 18 , and 24 , respectively.
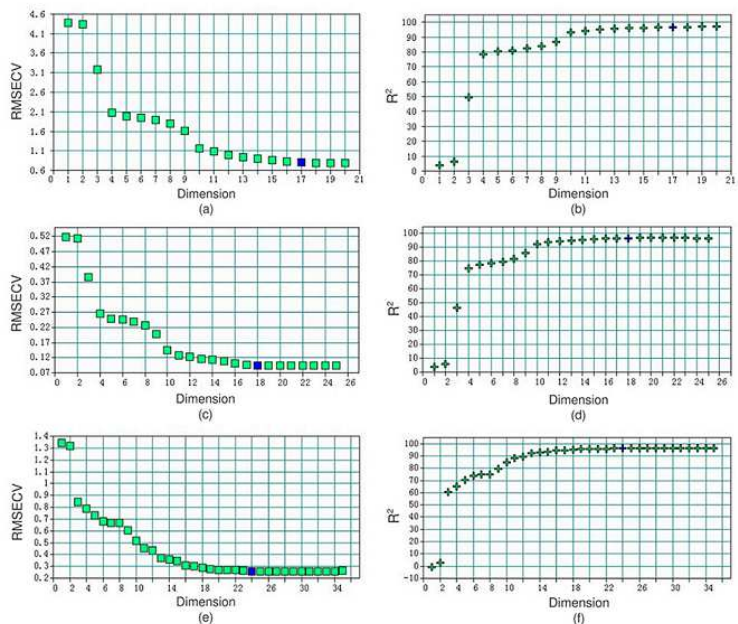

Figure 2: Dimensions of partial least squares factors. RMSECV versus dimension for (a) ginsenoside Rg1, (c) ginsenoside $R e$, and (e) notoginsenoside $R 1 ; R^{2}$ versus dimension for (b) ginsenoside $\mathrm{Rg} 1$, (d) ginsenoside $\mathrm{Re}$, and (f) notoginsenoside R1

\section{Predicted result of the validation set}

The calibration models were established using the PLS method with the obtained optimal parameters. Then the models were used to predict the values of ginsenoside Rg1, ginsenoside $\mathrm{Re}$, and notoginsenoside $\mathrm{R} 1$ in the $5^{\text {th }}$ and $10^{\text {th }}$ batch of samples. Figure 3 shows there is no significant difference between the values predicted by the NIR models and the values directly measured by HPLC. Furthermore, the results by NIR were obtained in $20 \mathrm{~s}$, but HPLC required at least $30 \mathrm{~min}$. Thus, the established models could satisfy the precision requirement of the online analysis for percolation extraction process of PTS. The parameters of the three optimal models are shown in Table 4.
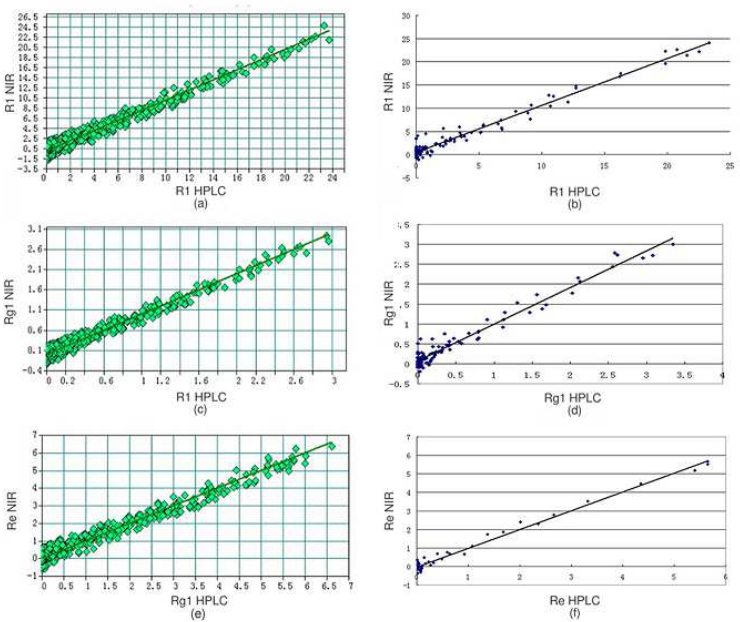

Figure 3: Correlation between values obtained using near-infrared (NIR) and HPLC. Correlation values for (a) notoginsenoside R1, (c) ginsenoside Rg1, and (e) ginsenoside $\mathrm{Re}$ of the calibration set; Correlation between values by NIRs and HPLC of (b) notoginsenoside R1, (d) ginsenoside Rg1, and (f) ginsenoside $R e$ of the validation set. 
Table 4: Parameters of optimal models for three active ingredients

\begin{tabular}{|c|c|c|c|c|}
\hline Variable & & Ginsenoside Rg1 & Ginsenoside Re & Notoginsenoside R1 \\
\hline Pretreatment & & MMN & MMN & MMN \\
\hline Wavelength & & $6475.9-5449.9$ & $6475.9-5449.9$ & $7501.9-5449.9$ \\
\hline$\left(\mathrm{cm}^{-1}\right)$ & & $4601.4-4246.5$ & $4601.4-4224$ & $4601.4-4246.5$ \\
\hline & Calibration & 336 & 336 & 336 \\
\hline Samples & Validation & 84 & 84 & 84 \\
\hline D & & 17 & 18 & 24 \\
\hline RMSECV $(\mathrm{mg} / \mathrm{mL})$ & & 0.798 & 0.095 & 0.259 \\
\hline$R^{2}$ (calibration) & & 0.9682 & 0.9681 & 0.9626 \\
\hline RMSEP $(\mathrm{ma} / \mathrm{mL})$ & & 1.110 & 0.496 & 0.390 \\
\hline$R^{2}$ (validation) & & 0.9831 & 0.9198 & 0.9661 \\
\hline
\end{tabular}

MMN: min-max normalization; RMSECV: root mean square errors of cross-validation; RMSEP: root mean square errors of prediction; $\mathrm{D}$ : dimensions

\section{DISCUSSION}

NIR technology is a useful tool for qualitative and quantitative analysis in many different areas, such as agriculture [12,13], food [14,15], and pharmaceutical industries. It is non-polluting, non-destructive, and provides real-time analysis and high accuracy [16-18]. NIR light is an electromagnetic wave between ultraviolet visible light and mid-infrared light in the wavelength range of $780-2526 \mathrm{~nm}$. The most prominent absorption bands of NIR are overtones and combinations relating to $-\mathrm{CH},-\mathrm{NH}$, and $-\mathrm{OH}$ (and $-\mathrm{SH}$ ) functional groups $[19,20]$. NIR spectroscope (NIRs) principles are intrinsically different from traditional analytical techniques, such as simple or no sample preparation, rapid measurement and non-destructive testing $[21,22]$. Based on these advantages, the combination of NIR with chemometric techniques has been applied to analysis of complex samples or ingredients including TCM $[23,24]$.

To find meaningful associations from NIRs, appropriate mathematical models need to be established. Recently, some chemometrics methods were used to establish NIR mathematical models, such as partial least squares (PLS), artificial neural networks (ANN), and multiple linear regression. Among these methods, PLS is the most popular because it can reveal information for the dependent variable as well as reduce the dimensions of the spectral matrix [25-27]. In the present study, NIR coupled with PLS was employed, and a mathematical model was successfully established for online monitoring of PTS (ginsenoside Rg1, ginsenoside $R e$, and notoginsenoside R1) in the percolation extraction process.

\section{CONCLUSION}

An online monitoring model has been successfully established for the percolation extraction process of PTS extracted from notoginseng using NIR technology combined with PLS. Overall, the proposed method and models are efficient and accurate, and can be applied for rapid analysis and online quality control in PTS manufacturing.

\section{DECLARATIONS}

\section{Acknowledgement}

This work was supported by Science and technology development foundation of Chengdu University of Traditional Chinese Medicine (no. YYZX1711, 2017) and Chinese High Technology of National Development and Reform Commission (No. 51, 2011).

\section{Conflict of interest}

No conflict of interest is associated with this work.

\section{Contribution of authors}

We declare that this work was done by the authors named in this article and all liabilities pertaining to claims relating to the content of this article will be borne by them. In addition, Guangfu Feng conceived and designed the study. Ruixue Zhong, Hui-min Wen, Xiao-yun Duan, Xiaoling Wang and Chuan-hong Wu performed the detail experiments. Yu-jie Liu, Jun Wan, Li-yang zhu, Yun-bei Chen and Ting-sen Wei collected and analyzed the data. Rui-xue Zhong and Yu-jie Liu wrote the manuscript. All authors have read and approved the manuscript for publication must be specified.

\section{REFERENCES}

1. Jiang $Y$, David B, Tu $P$, Barbin Y. Recent analytical approaches in quality control of traditional Chinese medicines--a review. Anal Chim Acta 2010; 657 (1): 918.

Trop J Pharm Res, October 2018; 17(10):2059 
2. Zhang $L, M a L$, Feng $W$, Zhang $C$, Sheng $F$, Zhang $Y, X u$ $C$, Dong $G$, Dong $X$, Xiao $X$, et al. Quality fluctuation detection of an herbal injection based on biological fingerprint combined with chemical fingerprint. Anal Bioanal Chem 2014; 406 (20): 5009-5018.

3. Ren $Y$, Zhang $P$, Yan $D$, Wang J, Du X, Xiao X. A strategy for the detection of quality fluctuation of a Chinese herbal injection based on chemical fingerprinting combined with biological fingerprinting. J Pharm Biomed Anal 2011; 56 (2): 436-442.

4. Editorial Committee of Chinese Pharmacopoeia. Chinese pharmacopoeia. Vol. 1. China Chemical Industry Press: Beijing; 2015; $p 58$.

5. Ng TB. Pharmacological activity of sanchi ginseng (Panax notoginseng). J Pharm Pharmacol 2006; 58:1007-1019.

6. Guo HB, Cui XM, An N, Cai GP. Sanchi ginseng (Panax notoginseng (Burkill) F. H. Chen) in China: distribution, cultivation and variations. Genet Resour Crop Ev 2010; 57 (3): 453-460.

7. Xia P, Guo H, Liang Z, Cui X, Liu Y, Liu F. Nutritional composition of Sanchi (Panax notoginseng) seed and its potential for industrial use. Genet Resour Crop Ev 2014, 61(3): 663-667.

8. Luo H. Analysis of the transcriptome of Panax notoginseng root uncovers putative triterpene saponinbiosynthetic genes and genetic markers. Bmc Genomics 2011; 12(Supp/ 5): S5-S5.

9. Wan JB, Lai CM, Li SP, Lee MY, Kong LY, Wang YT. Simultaneous determination of nine saponins from Panax notoginseng, using HPLC and pressurized liquid extraction [J]. J Pharm Biomed Anal 2006; 41(1): 274 279.

10. Ma T, Huang C, Meng $X$, Zhang $Q$, Zhang $L, L v X$, Jin $Y$, $X i e \mathrm{~J}, \mathrm{Li} \mathrm{J}$. Fingerprint analysis of Hawk-tea by high performance liquid chromatography. Food Chem 2011; 129: 551-556.

11. Li SP, Qiao CF, Chen YW, Zhao J, Cui XM, Zhang QW. A novel strategy with standardized reference extract qualification and single compound quantitative evaluation for quality control of Panax notoginseng used as a functional food. J Chromatogr A 2013; 1313(19): 302-307.

12. Zhao LL, Xi L, Miao ZH, Guo HX, Wang JAH, Lei ML. Antioxidant activity of bamboo-leaf extracts from the species Dendrocalamopsis Oldhami. Sci Res Essays 2012; 7(44): 3789-3796.

13. Zheng $Y$, Gao $P$, Wu Z, Chen, L, Wu D. Effect of Fertilization on nutrient components of bamboo shoot and leaves nutrient status shoot emergence stages in Dendrocalamopsis oldhami. Scientia Silvae Sinicae 2004; 40(6): 79-84.

14. Menesatti $P$, Pallottino F, Antonucci $F$, Roccuzzo G, Intrigliolo F, Costa C. Non-destructive Proximal Sensing for Early Detection of Citrus Nutrient and Water Stress. Springer Netherlands, 2012:113-123.
15. Sánchez MT, Haba MJDL, Serrano I, Pérez-Marín D. Application of NIRS for Nondestructive Measurement of Quality Parameters in Intact Oranges During On-Tree Ripening and at Harvest. Food Anal Method, 2013; 6(3): 826-837.

16. Vakili H, Nyman JO, Genina N, Preis M, Sandler N. Application of a colorimetric technique in quality control for printed pediatric orodispersible drug delivery systems containing propranolol hydrochloride. Int J Pharm 2016; 511(1): 606-618.

17. Bhaskar DK. Formulation and evaluation of orodispersible tablets of propranolol hydrochloride. Int $J$ Pharm Res \& Dev 2011; 2(12).

18. Vakili $H$, Wickström $H$, Desai $D$, Preis $M$, Sandler $N$. Application of a handheld NIR spectrometer in prediction of drug content in inkjet printed orodispersible formulations containing prednisolone and levothyroxine. Int J Pharm 2017; 524(1-2): 414-423.

19. Luypaert J, Massart DL, Heyden YV. Near-infrared spectroscopy applications in pharmaceutical analysis. Talanta 2007; 72(3): 865-883.

20. Ruan L. The principle and application of near-infrared spectroscopy for the Chinese herbal medicines. Chin J Pharm Anal 2011; 31(2): 408-417.

21. Li W, Xing L, Cai $Y, Q u H$. Classification and quantification analysis of Radix Scutellariae from different origins with near infrared diffuse reflection spectroscopy. Vib Spectros 2011; 55(1):58-64.

22. Liu W, Wang ZZ, Qing JP, Li HJ, Xiao W. Classification and quantification analysis of peach kernel from different origins with near-infrared diffuse reflection spectroscopy. [J]. Pharmacogn Mag 2014, 10(40): 441-448.

23. Huang M, Kim MS, Delwiche SR, Chao K, Qin J, Mo C. Quantitative analysis of melamine in milk powders using near-infrared hyperspectral imaging and band ratio[J]. $J$ Food Eng 2016; 181:10-19.

24. Li GZ, Xin Z, Li JN, Zhi-Bin, Xin, G., Shi XH. Rapid identification of adulterated cow milk by non-linear pattern recognition methods based on near infrared spectroscopy. Food Chem, 2014, 145(4): 342.

25. Kasemsumran S, Du YP, Maruo K, Ozaki Y. Improvement of partial least squares models for in vitro and in vivo glucose quantifications by using nearinfrared spectroscopy and searching combination moving window partial least squares. Chemometr Intell Lab 2006; 82(1-2): 97-103.

26. Igne B, Reeves JBI, Mccarty G, Hively WD, Lund E, Hurburgh CRJ. Evaluation of spectral pretreatments, partial least squares, least squares support vector machines and locally weighted regression for quantitative spectroscopic analysis of soils. I Near Infrared Sp 2010; 18(3): 167.

27. Reeves IJ. The Effect of Spectral Pretreatments on the PIs Modeling of Agricultural Products. Near Infrared $S p$ 2004; 12(1): 177. 